\title{
Efficacy and Safety of DWJ1252 Compared With Gasmotin in the Treatment of Functional Dyspepsia: A Multicenter, Randomized, Double-blind, Active-controlled Study
}

\begin{abstract}
Jin Hwa Park, ${ }^{1}$ Kang Nyeong Lee, ${ }^{1}$ Oh Young Lee, ${ }^{1 *}$ Myung-Gyu Choi, ${ }^{2}$ Hyunsoo Chung, ${ }^{3}$ Suck-Chei Choi, ${ }^{4}$ Nayoung Kim, ${ }^{5}$ Hyojin Park, ${ }^{6}$ In-Kyung Sung, ${ }^{7}$ Chong II Sohn, ${ }^{8}$ Sam Ryong Jee, ${ }^{9}$ Jae Young Jang, ${ }^{10}$ Poong-Lyul Rhee, ${ }^{11}$ Moo In Park, Joong Goo Kwon, ${ }^{13}$ Kyung Sik Park, ${ }^{14}$ Kwang Jae Lee, ${ }^{15}$ and Joon Seong Lee ${ }^{16}$

${ }^{1}$ Department of Internal Medicine, Hanyang University College of Medicine, Seoul, Korea; ${ }^{2}$ Department of Internal Medicine, Catholic University College of Medicine, Seoul, Korea; ${ }^{3}$ Department of Internal Medicine, Seoul National University, Seoul, Korea; ${ }^{4}$ Department of Internal Medicine, Wonkwang University College of Medicine, Iksan, Jeollabuk-do, Korea; ${ }^{5}$ Department of Internal Medicine, Seoul National University Bundang Hospital, Seongnam, Gyeonggi-do, Korea; ${ }^{6}$ Department of Internal Medicine, Yonsei University Gangnam Hospital, Seoul, Korea; ${ }^{7}$ Department of Internal Medicine, Konkuk University College of Medicine, Seoul, Korea, ${ }^{8}$ Department of Internal Medicine, Kangbuk Samsung Medical Center, Sungkyunkwan University School of Medicine, Seoul, Korea; ${ }^{9}$ Department of Internal Medicine, Inje University College of Medicine, Busan, Korea; ${ }^{10}$ Department of Internal Medicine, Kyunghee University College of Medicine, Seoul, Korea; ${ }^{11}$ Department of Internal Medicine, Samsung Medical Center, Sungkyunkwan University School of Medicine, Seoul, Korea; ${ }^{12}$ Department of Internal Medicine, Kosin University College of Medicine, Busan, Korea; ${ }^{13}$ Department of Internal Medicine, Daegu Catholic University School of Medicine, Daegu, Korea; ${ }^{14}$ Department of Internal Medicine, Keimyung University College of Medicine, Daegu, Korea; ${ }^{15}$ Department of Internal Medicine, Ajou University College of Medicine, Suwon, Gyeonggi-do, Korea; and ${ }^{16}$ Department of Internal Medicine, Soonchunhyang University College of Medicine, Seoul, Korea
\end{abstract}

\section{Background/Aims}

Prokinetics such as mosapride citrate CR (conventional-release; Gasmotin) are commonly used in functional dyspepsia (FD). This study aims to evaluate the efficacy and safety of once-a-day mosapride citrate SR (DWJ1252), a sustained-release formulation of mosapride citrate, compared with mosapride citrate CR 3 times a day, in patients with FD.

\section{Methods}

In this multicenter, randomized, double-blind, active-controlled, non-inferiority study, 119 patients with FD (by the Rome III criteria, 60 for mosapride citrate SR and 59 for mosapride citrate (R) were randomly allocated to mosapride citrate SR once daily or mosapride citrate CR thrice daily for 4 weeks in 16 medical institutions. Primary end point was the change in gastrointestinal symptom (GIS) score from baseline, assessed by GIS questionnaires on 5-point Likert scale after 4-week treatment. Secondary end points and safety profiles were also analyzed.

\section{Results}

The study included 51 and 49 subjects in the mosapride citrate SR and mosapride citrate CR groups, respectively. GIS scores at week 4 were significantly reduced in both groups (mean \pm SD: $-10.04 \pm 4.45$ and $-10.86 \pm 5.53$ in the mosapride citrate SR and mosapride citrate $C R$ groups, respectively; $P<0.001$ ), and the GIS changes from baseline did not differ between the 2 groups (difference, 0.82 point; $95 \% \mathrm{Cl},-1.17,2.81 ; P=0.643$ ). Changes in GIS at weeks 2 and 4 and quality of life at week 4 , and the improvement rates of global assessments at weeks 2 and 4, did not differ between the groups. Adverse events were similar in the 2 groups, and there were no serious adverse events.

\section{Conclusion}

In patients with FD, mosapride citrate SR once daily is as effective as mosapride citrate CR thrice daily, with a similar safety profile.

(J Neurogastroenterol Motil 2021;27:87-96)

Key Words

Dyspepsia; Gastrointestinal disease; Mosapride 
Received: March 31, 2020 Revised: May 22, 2020 Accepted: June 11, 2020

(a) This is an Open Access article distributed under the terms of the Creative Commons Attribution Non-Commercial License (http://creativecommons. org/licenses/by-nc/4.0) which permits unrestricted non-commercial use, distribution, and reproduction in any medium, provided the original work is properly cited.

*Correspondence: Oh Young Lee, MD, PhD

Department of Internal Medicine, Hanyang University Hospital, 222, Wangsimni-ro, Seongdong-gu, Seoul 133-791, Korea Tel: +82-2-2290-8343, Fax: +82-2-2298-8314, E-mail: leeoy@hanyang.ac.kr

\section{Introduction}

Patients with dyspepsia complain of early satiety, postprandial fullness, and epigastric pain/soreness, which are symptoms thought to originate from the gastroduodenum. The global prevalence of dyspepsia is reported to be about $20.0 \%$; however, in up to $70.0 \%$ of those who complain of dyspepsia there is no known organic cause. ${ }^{1}$ These patients can be diagnosed with functional dyspepsia (FD) based on the Rome III criteria. The symptoms of FD may be due to multiple factors including visceral hypersensitivity, impaired gastroduodenal motility, autonomic nervous system dysfunction, altered microbiota, altered gut-brain axis, and psychological problems, but the exact mechanism of the symptoms seen in patients with FD is still being investigated. Therefore, no definitive treatment for FD has not yet been established, and instead empirical treatments are widely used; these include acid suppressive drugs, prokinetics, pain modulators, and psychotherapy. ${ }^{2}$

The empirical treatment agents for FD are prokinetics such as metoclopramide, domperidone, cisapride, tegaserod, and mosapride citrate. In a meta-analysis of 1844 patients with $\mathrm{FD}$, prokinetics gave an approximately $30.0 \%$ higher response rate than placebo. ${ }^{3}$ Furthermore, a recent meta-analysis of 29 trials comparing with placebo found an average percentage symptom improvement of $40.0 \%$ in the prokinetic group, compared to $26.1 \%$ in the placebo group. ${ }^{4}$ Prokinetics may improve FD symptoms by affecting gastric accommodation or increasing gastroduodenal contraction, thereby shortening gastric emptying time. Among these prokinetics, mosapride citrate exerts its prokinetic effect by releasing acetylcholine through selectively activating 5 -hytroxytryptamine 4 receptors thereby causing contraction of smooth muscle fibers in the gastroduodenum. ${ }^{5,6}$ In various experimental and clinical studies, mosapride induced increased gastric emptying and showed efficacy and safety in patients with $\mathrm{FD}^{7,8}$ Also, the Korean national clinical guidelines for $\mathrm{FD}$ recommend mosapride and other prokinetics for patients with FD. ${ }^{9}$

However, the symptoms of most FD patients become chronic and inconsistent with time. As a result, mosapride citrate CR (conventional-release; Gasmotin) needs to be administered 3 times per day, leading to problems of low compliance due to discomfort. In contrast to mosapride citrate CR, mosapride citrate SR (DWJ1252) is a sustained release formulation of mosapride citrate that is released at a constant rate over the whole day. Therefore, once-daily dosing with mosapride citrate SR displays sustained efficacy from day to night similar to mosapride citrate CR 3 times daily. However, it is not known whether mosapride citrate SR has similar efficacy and safety to mosapride citrate CR 3 times daily in FD. This clinical trial was conducted to test the non-inferiority of mosapride citrate SR (mosapride citrate salt $15 \mathrm{mg}$ ) administered once daily for 4 weeks to mosapride citrate CR (mosapride citrate salt $5 \mathrm{mg}$ ) 3 times daily in terms of efficacy and safety in patients with $\mathrm{FD}$ (diagnosed based on the Rome III criteria).

\section{Materials and Methods}

\section{Subjects}

Eligible patients were male and female adults of $\geq 19$ years, with a history of FD symptoms (early satiety, postprandial fullness, epigastric pain, and epigastric soreness) for at least 6 months before screening, sustained over the previous 3 months, without any organic disorders affecting dyspepsia at screening endoscopy (according to the Rome III criteria for FD). Patients had to have at least 1 of the above 4 symptoms of more than moderate severity and at least 3 symptoms of more than moderate severity out of the 10 symptoms: nausea, vomiting, bloating, abdominal cramps, early satiety, acid eructation/heartburn, sickness, loss of appetite, retrosternal discomfort, and epigastric pain. ${ }^{10}$

Exclusion criteria were: history of any surgery affecting gastrointestinal (GI) motility, GI bleeding, mechanical obstruction, or perforation; irritable bowel syndrome, inflammatory bowel disease, gastroesophageal reflux disease, peptic ulcer disease, pancreatic disorders, GI malignancy, any malignancy within 5 years; an organic cause of gastroparesis; significant morbidity of heart, kidney, liver, or lung; neuropsychiatric disorder such as major depressive disorder or anxiety disorder, or history of drug abuse; uncontrolled diabetes mellitus or hypertension; abnormal alanine aminotransferase/aspartate transaminase ( $>3$ times the normal upper limit) or creatinine clearance $(<30 \mathrm{~mL} / \mathrm{min})$. Also excluded were any 
patients who took medication to influence the outcome assessment (acid suppressive drugs, other prokinetics, mucosal protectants, fundic relaxants, and cholinergic/anti-cholinergic agents). Medications such as prokinetics, antisecretory agents, mucosal protectants, fundic relaxants, cholinergic agents, antipsychotic drugs, NSAIDs/ aspirin, erythromycin, and steroids as well as Helicobacter pylori eradication therapy were forbidden.

\section{Study Design}

This was a multicenter, randomized, double-blind, activecontrolled, non-inferiority study performed from December 2016 to April 2017 in 16 Korean referral hospitals (ClinicalTrial.gov Identifier: NCT03024086). It was conducted according to the ethical principles based on the Declaration of Helsinki and good clinical practice, as well as the requirements of the Ministry of Food and Drug Safety.

The study protocol was reviewed and approved by the institutional review board of each hospital as follows: Hanyang University College of Medicine (HYUH 2016-09-023), Catholic University College of Medicine (KC16MDMT0808), Seoul National University Hospital (H-1610-074-800), Wonkwang University College of Medicine (WKUH 201610-CTDG-021), Seoul National University Bundang Hospital (B-1610/366-008), Yonsei University Gangnam Hospital (3-2016-0269), Konkuk University College of Medicine (KUH1010819), Kangbuk Samsung Medical Center, Sungkyunkwan University School of Medicine (KBSMC 201610-003), Inje University College of Medicine (16-0220), Kyunghee University College of Medicine (KHUH 2016-10-018), Samsung Medical Center, Sungkyunkwan University School of Medicine (2016-09-124), Kosin University College of Medicine (KUCH 2016-09-027), Daegu Catholic University School of Medicine (16153), Keimyung University College of Medicine (DSMC 201609-039), Ajou University College of Medicine (AJIRB-MEDCT3-16-366), Soonchunhyang University College of Medicine (2016-10-003). All patients were provided to written informed consent before enrolment.

For a run-in period, eligible patients were randomly allocated to the mosapride citrate SR (mosapride citrate SR + mosapride citrate $\mathrm{CR}$ placebo) or mosapride citrate CR (mosapride citrate
$\mathrm{SR}$ placebo + mosapride citrate $\mathrm{CR}$ ) group for 4 weeks in 16 medical institutions. Treatments were assigned by a stratified block randomization method designed to allocate equal numbers of patients to the 2 treatment arms. To maintain double blind conditions, participants took identically-appearing tablets 3 times daily before meals ( 2 pills in the morning and 1 pill each for lunch and dinner) (Table 1). The 2 pills in the morning consisted of mosapride citrate $\mathrm{SR}+$ mosapride citrate CR placebo for the study subjects and mosapride citrate SR + mosapride citrate CR for the controls, respectively. Mosapride citrate $\mathrm{SR}$ is a sustained-release formulation containing $15 \mathrm{mg}$ of mosapride citrate anhydride per tablet and is taken once daily. The control drug, mosapride citrate $\mathrm{CR}$, contains $5 \mathrm{mg}$ of mosapride citrate anhydride and is taken 3 times a day. In a previous study comparing the pharmacodynamic characteristics of mosapride citrate SR and mosapride citrate $\mathrm{CR}$, the areas under the curve of blood levels-time were log 0.89-1.17, indicating that there is biological equivalence between the 2 drugs. Therefore, mosapride citrate $\mathrm{CR}$ was considered to be a suitable control agent for noninferiority testing.

Follow-up visits during the study period to assess gastrointestinal symptom (GIS) scores, dyspepsia-specific quality of life (QoL), drug compliance, and adverse events were scheduled at weeks 2 and 4 (Fig. 1). Compliance with medication was confirmed at Visit 2 and Visit 4 by patients returning the unused portion of the medication. The compliances with the test medication (or test drug placebo) and control medication (or the placebo of the control medication) were calculated, and the averages of the 2 were taken as the final compliance with medication. Dyspepsia-specific QoL and $H$. pylori status were assessed using Nepean Dyspepsia IndexKorean version (NDI-K) ${ }^{11}$ and the urea breath test, respectively.

\section{Study Assessments}

\section{Efficacy}

The primary endpoint was the change in GIS scores from baseline assessed by symptom questionnaires after 4 weeks of treatment. Subjects completed GIS questionnaires to evaluate the severity of 10 dyspeptic symptoms, nausea, vomiting, bloating, abdominal cramps, early satiety, acid eructation/heartburn, sickness, loss of ap-

Table 1. Method of Administering the Drugs in This Clinical Trial

\begin{tabular}{ccc}
\hline Group & Methods of administering the drug & Morning \\
\hline Mosapride citrate SR group & Mosapride citrate SR + Mosapride citrate CR placebo & Dinner \\
Mosapride citrate CR group & Mosapride citrate SR placebo + Mosapride citrate CR & $\checkmark$ \\
\hline
\end{tabular}

, mosapride citrate SR; mosapride citrate $\mathrm{CR}$; $\bigcirc$, mosapride citrate SR placebo; $\diamond$, mosapride citrated CR placebo. 


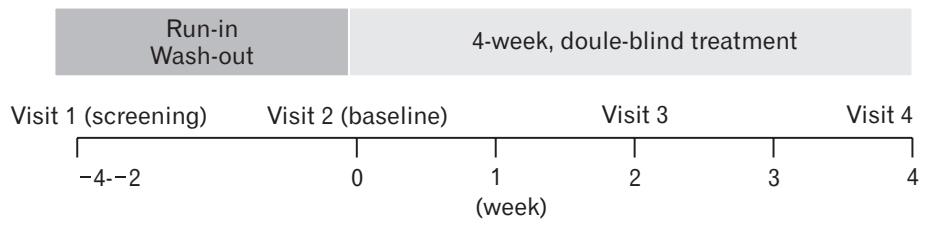

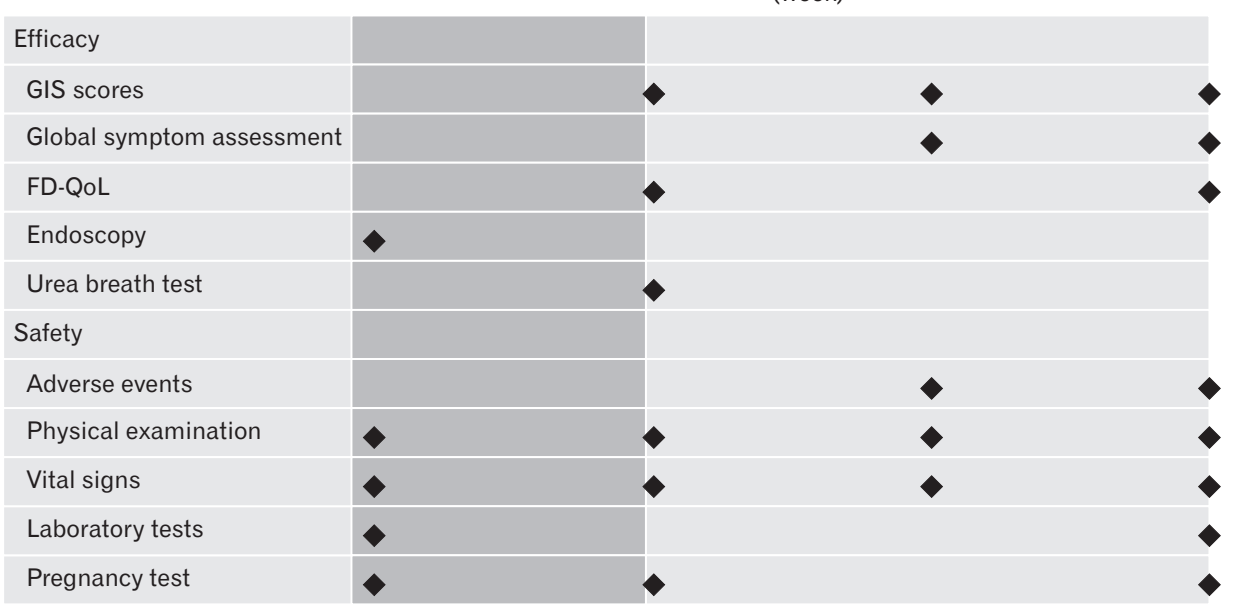

Figure 1. The design of the study. GIS, gastrointestinal symptom scores; FDQoL, functional dyspepsia-related quality of life.

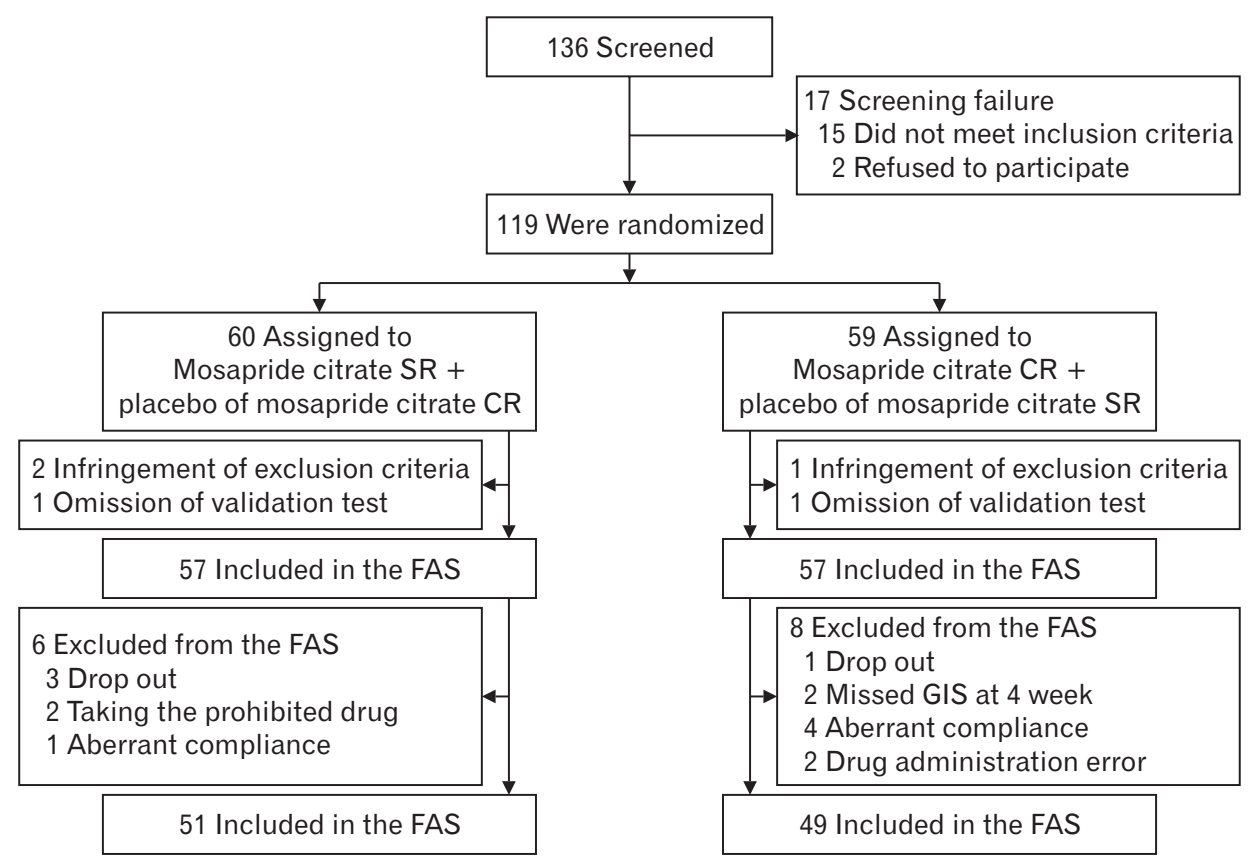

Figure 2. Flow sheet of the study. FAS, full analysis set; GIS, gastrointestinal symptom; PPS, per-protocol set.

petite, retrosternal discomfort, and epigastric pain on 5-point Likert scales ( 0 , symptom-free; 1 , mild; 2 , moderate; 3 , severe; 4 , very severe) at 0 (baseline), 2 , and 4 weeks of treatment). Total GIS scores were the sum of the individual symptom severity scores.

The secondary endpoints were the changes in GIS scores from baseline at week 2, the changes in GIS scores for each symptom from baseline at weeks 2 and 4, the improvement rate of global assessments at weeks 2 and 4, and the changes in total and areaspecific dyspepsia-specific QoL scores from baseline at week 4. Symptom improvements were classified as loss of symptoms, markedly improved, slightly improved, no change, and aggravated; "loss of symptoms" and "markedly improved" were together defined as "improvement." Dyspepsia-specific QoL was assessed in areas of tension/sleep, interference with daily activities, eating/drinking, 
knowledge/control, and work/study by the NDI-K.

Exploratory endpoints included all the primary and secondary endpoints according to $H$. pylori infection status and FD subtype (postprandial distress syndrome [PDS] and epigastric pain syndrome), respectively.

\section{Safety}

The safety of study drugs and compliance were assessed based on adverse events reported by subjects, physical examination, and laboratory tests, at each institution at weeks 2 and 4 after randomization. Drug compliance was considered good when the compliance was $>80.0 \%$ and $<120.0 \%$. The safety set composed of all subjects who took the study drug at least once after randomization and underwent follow-up for safety assessment.

\section{Statistical Methods}

Data were analyzed by 2-tailed tests with significance set at $P<0.05$, using SAS version 9.3 (SAS Institute, Cary, NC, USA). Continuous variables are presented as numbers, means, standard deviation, minimum, median, and maximum. After normality testing, continuous variables were analyzed by paired $t$ tests or Wilcoxon signed rank tests for in-group comparisons or by 2 sample $t$ tests or Wilcoxon rank sum tests for inter-group comparisons. Categorical variables were analyzed by McNemar's tests for in-group comparisons and by Pearson's chi-square tests or Fisher's exact tests for inter-group comparisons.

Safety set analysis was performed in all patients who received at least one dose of test drug and had follow-up assessment. Analyses of demographics and baseline characteristics were performed in all patients of each group included in the full analysis set (FAS). Efficacy endpoints were analyzed in the per-protocol set population, defined as the subset of the FAS population excluding those with major protocol violations.

In previous studies, changes in GIS scores were $-12.1--5.6$ and $-3.0--1.5$ in the mosapride citrate SR and mosapride citrate CR groups, respectively. Given that the weighted averages of GIS changes in the mosapride citrate SR and mosapride citrate CR groups were -9.08 and -2.28 , respectively, ${ }^{12-14}$ the margin of noninferiority was conservatively set at 3.0 , approximately half of the statistical tolerance limit $([-9.08--2.28] / 2=3.4)$. With the assumption of non-inferiority, 49 patients were needed in each group for a one-sided test of significance level $2.5 \%$, power $80.0 \%, 1: 1$ assignment. The sample size was estimated from a previous study: assuming a $5.0 \%$ significance level, $80.0 \%$ test power, and $20.0 \%$ drop-out rate, a total of 124 patients was needed (62 per group).

\section{Results}

\section{Subjects}

Of the 136 patients screened, 17 were excluded, and the remaining 119 were randomly assigned, with 60 in the mosapride citrate $\mathrm{SR}$ and 59 in the mosapride citrate CR group (Fig. 2). A total of 114 patients were included in the FAS (57 each in the mosapride citrate SR and mosapride citrate CR groups). Of those, 51 and 49 were included in the per-protocol set. There were no differences in baseline characteristics between the groups (Table 2).

\section{Efficacy}

\section{Primary efficacy variable}

Changes in GIS scores at week 4 from baseline were not different

Table 2. Demographic and Baseline Characteristics of the Subjects

\begin{tabular}{cccc}
\hline Baseline & $\begin{array}{c}\text { Mosapride citrate } \\
\text { SR group } \\
\text { characteristics }\end{array}$ & $\begin{array}{c}\text { Mosapride citrate } \\
\text { CR group } \\
(\mathrm{n}=57)\end{array}$ & $P$-value \\
\hline Age $(\mathrm{yr})$ & & & \\
Mean $\pm \mathrm{SD}$ & $44.37 \pm 14.58$ & $43.12 \pm 14.64$ & $0.753^{\mathrm{a}}$ \\
Median & 42.00 & 39.00 & \\
Min, Max & $22.00,68.00$ & $23.00,68.00$ & \\
Age groups $(\mathrm{yr})$ & & & \\
$<$ 65 & $53(93.0 \%)$ & $54(94.7 \%)$ & $>0.999^{\mathrm{b}}$ \\
$\geq 65$ & $4(7.0 \%)$ & $3(5.3 \%)$ & \\
Sex & & & \\
Male & 9 & 7 & $0.589^{\mathrm{c}}$ \\
Female & 48 & 50 & \\
Height $(\mathrm{cm})$ & & & \\
Mean $\pm \mathrm{SD}$ & $161.96 \pm 9.04$ & $161.27 \pm 6.44$ & $0.744^{\mathrm{a}}$ \\
Median & 161.50 & 161.70 & \\
Min, Max & $148.00,184.00$ & $141.00,173.00$ & \\
Weight $(\mathrm{kg})$ & & & $0.088^{\mathrm{a}}$ \\
Mean $\pm \mathrm{SD}$ & $60.53 \pm 10.96$ & $57.84 \pm 9.14$ & $0.210^{\mathrm{a}}$ \\
Median & 58.20 & 57.00 & \\
Min, Max & $42.00,92.80$ & $42.40,92.70$ & \\
BMI $\left(\mathrm{kg} / \mathrm{m}^{2}\right)$ & & & \\
Mean $\pm \mathrm{SD}$ & $22.96 \pm 2.69$ & $22.21 \pm 3.04$ & \\
Median & 23.27 & 21.53 & \\
Min, Max & $18.18,28.59$ & $17.37,34.89$ & \\
\hline
\end{tabular}

BMI, body mass index.

${ }^{a}$ Wilcoxon rank sum test.

${ }^{6}$ Fisher's exact test.

'Pearson's chi-square test . 
between the groups. The mean changes in GIS scores from baseline were $-10.04 \pm 4.45$ in the mosapride citrate SR group and -10.86 \pm 5.53 in the mosapride citrate CR group, respectively, demonstrating a significant improvement of GIS in both groups $(P<0.001)$ (Fig. 3). The difference in mean GIS changes between the 2 groups was 0.82 (95\% CI, $-1.17,2.81)$, the upper limit of the $97.5 \% \mathrm{CI}$ in the one-sided comparison was 2.81, which was below the margin of inferiority of 3.0, indicating that mosapride citrate SR once daily is not inferior to mosapride citrate CR 3 times daily (Table 3).

\section{Secondary efficacy variables}

Most of the secondary efficacy variables did not differ between the mosapride citrate SR and mosapride citrate CR groups. Changes in GIS scores at week 2 from baseline were not different between

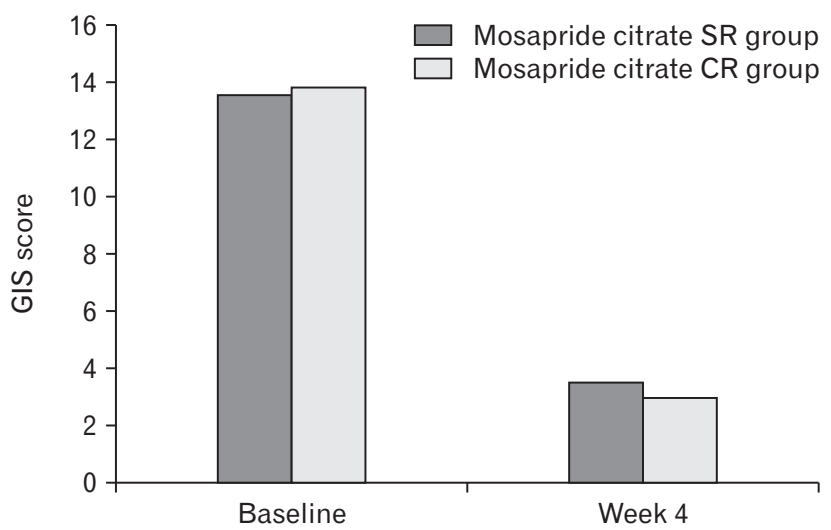

Figure 3. Summary of the primary endpoint (gastrointestinal symptom [GIS] scores at baseline and week 4 in the per-protocol set population). the 2 groups. GIS scores at week 2 were significantly reduced in both groups $(-6.78 \pm 5.32$ and $-9.16 \pm 5.13 ; P<0.001$ ) (Table 4). GIS changes from baseline at weeks 2 and 4 for symptoms of nausea, vomiting, bloating, abdominal cramps, early satiety, acid eructation/heartburn, sickness, loss of appetite, retrosternal discomfort, and epigastric pain, decreased significantly, while the changes in GIS scores for distension at week $2(P=0.006)$ and for early satiety at weeks $2(P=0.001)$ and $4(P=0.024)$ were greater in the mosapride citrate $\mathrm{CR}$ group than the mosapride citrate $\mathrm{SR}$ group.

The improvement rates in global assessment at weeks 2 and

Table 4. Changes in Gastrointestinal Symptom Scores From Baseline at Week 2

\begin{tabular}{lccc}
\hline \multicolumn{1}{c}{ GIS } & $\begin{array}{c}\text { Mosapride citrate } \\
\text { SR group }\end{array}$ & $\begin{array}{c}\text { Mosapride citrate } \\
\text { CR group }\end{array}$ & $P$-value \\
& $\mathrm{n}=51$ & $\mathrm{n}=49$ & \\
PPS & $13.55 \pm 5.21$ & $13.84 \pm 5.32$ & $0.721^{\mathrm{b}}$ \\
Baseline & $6.76 \pm 6.09$ & $4.67 \pm 3.69$ & \\
Week 2 & $-6.78 \pm 5.32$ & $-9.16 \pm 5.13$ & $0.059^{\mathrm{b}}$ \\
Change $^{\mathrm{a}}$ & $<0.001^{\mathrm{c}}$ & $<0.001^{\mathrm{d}}$ & \\
In group $P$-value & $\mathrm{n}=57$ & $\mathrm{n}=57$ & \\
FAS & $13.55 \pm 5.00$ & $14.07 \pm 5.58$ & $0.541^{\mathrm{b}}$ \\
Baseline & $6.66 \pm 5.91$ & $5.33 \pm 5.38$ & \\
Week 2 $_{\text {Change }}^{\mathrm{a}}$ & $-6.66 \pm 5.20$ & $-8.74 \pm 5.66$ & $0.055^{\mathrm{b}}$ \\
In group $P$-value & $<0.001^{\mathrm{c}}$ & $<0.001^{\mathrm{d}}$ & \\
\hline
\end{tabular}

GIS, gastrointestinal symptom scores; PPS, per-protocol set; FAS, full analysis set.

${ }^{a}$ Changes in score related to symptoms (week 2-baseline).

${ }^{b}$ Wilcoxon rank sum test.

${ }^{c}$ Paired $t$ test.

${ }^{\mathrm{d}}$ Wilcoxon signed rank test.

Table 3. Changes in Gastrointestinal Symptom Scores From Baseline at Week 4

\begin{tabular}{|c|c|c|c|c|}
\hline GIS & Mosapride citrate SR group & Mosapride citrate CR group & Difference $[95 \% \mathrm{CI}]^{\mathrm{a}}$ & $P$-value \\
\hline PPS & $\mathrm{n}=51$ & $\mathrm{n}=49$ & & \\
\hline Baseline & $13.55 \pm 5.21$ & $13.84 \pm 5.32$ & & $0.721^{\mathrm{c}}$ \\
\hline Week 4 & $3.51 \pm 3.85$ & $2.98 \pm 3.11$ & & \\
\hline Change $^{\mathrm{b}}$ & $-10.04 \pm 4.45$ & $-10.86 \pm 5.53$ & $0.82[-1.17,2.81]$ & $0.643^{\mathrm{c}}$ \\
\hline In group $P$-value & $<0.001^{\mathrm{d}}$ & $<0.001^{\mathrm{d}}$ & & \\
\hline FAS & $\mathrm{n}=57$ & $\mathrm{n}=57$ & & \\
\hline Baseline & $13.55 \pm 5.00$ & $14.07 \pm 5.58$ & & $0.541^{\mathrm{c}}$ \\
\hline Week 4 & $3.49 \pm 3.82$ & $3.70 \pm 5.22$ & & \\
\hline Change & $-9.86 \pm 4.48$ & $-10.37 \pm 6.23$ & $0.51[-1.51,2.52]$ & $0.820^{\mathrm{c}}$ \\
\hline In group $P$-value & $<0.001^{\mathrm{d}}$ & $<0.001^{\mathrm{d}}$ & & \\
\hline
\end{tabular}

GIS, gastrointestinal symptom scores; PPS, per-protocol set; FAS, full analysis set.

${ }^{a}$ Inter-group Difference $[95 \% \mathrm{CI}]=$ mosapride citrate SR-mosapride citrate CR 95\% CI based on $t$ test.

${ }^{\mathrm{b}}$ Changes in score related to symptoms (week 4-baseline).

${ }^{c}$ Wilcoxon rank sum test.

${ }^{\mathrm{d}}$ Wilcoxon signed rank test. 
4 were not different in the 2 groups and tended to increase from week 2 to week $4(27.5 \%$ [14/51] vs $38.8 \%$ [19/49], $P=0.229$ at week $2 ; 54.90 \%(28 / 51)$ vs $53.06 \%$ (26/49), $P=0.854$ at week 4$)$ (Supplementary Table 1).

Dyspepsia-specific QoL scores increased significantly in the 2 groups after 4 weeks of treatment (63.82 \pm 82.91 and 67.12 $\pm 81.11, P<0.001)$, with no difference between the 2 groups $(P$ $>0.999)$. Scores for QoL in the subscale of the NDI-K for ten-

Table 5. Changes in Dyspepsia-specific Quality of Life Scores From Baseline at Week 4

\begin{tabular}{|c|c|c|c|}
\hline $\begin{array}{l}\text { NDI-K } \\
\text { scores }\end{array}$ & $\begin{array}{l}\text { Mosapride citrate } \\
\text { SR group } \\
(\mathrm{n}=51)\end{array}$ & $\begin{array}{c}\text { Mosapride citrate } \\
\text { CR group } \\
(\mathrm{n}=49)\end{array}$ & $\begin{array}{c}\text { Intergroup } \\
P \text {-value }\end{array}$ \\
\hline \multicolumn{4}{|l|}{ Total } \\
\hline Baseline & $276.34 \pm 76.11$ & $288.57 \pm 70.79$ & $0.465^{\mathrm{b}}$ \\
\hline Week 4 & $340.16 \pm 80.48$ & $355.69 \pm 85.46$ & \\
\hline Change $^{a}$ & $63.82 \pm 82.91$ & $67.12 \pm 81.11$ & $>0.999^{\mathrm{b}}$ \\
\hline In group $P$-value & $<0.001^{\mathrm{c}}$ & $<0.001^{\mathrm{d}}$ & \\
\hline \multicolumn{4}{|l|}{ Tension/sleep } \\
\hline Baseline & $58.14 \pm 19.00$ & $60.22 \pm 17.16$ & $0.567^{\mathrm{e}}$ \\
\hline Week 4 & $73.38 \pm 17.55$ & $75.45 \pm 19.62$ & \\
\hline Change $^{a}$ & $15.23 \pm 20.27$ & $15.23 \pm 18.59$ & $0.890^{\mathrm{b}}$ \\
\hline In group $P$-value & $<0.001^{\mathrm{c}}$ & $<0.001^{\mathrm{d}}$ & \\
\hline \multicolumn{4}{|c|}{ Interference with daily activities } \\
\hline Baseline & $57.23 \pm 16.14$ & $58.43 \pm 17.56$ & $0.721^{\mathrm{e}}$ \\
\hline Week 4 & $65.61 \pm 18.38$ & $69.23 \pm 18.86$ & \\
\hline Change $^{\mathrm{a}}$ & $8.38 \pm 18.94$ & $10.79 \pm 19.38$ & $0.497^{\mathrm{b}}$ \\
\hline In group $P$-value & $<0.001^{\mathrm{c}}$ & $<0.001^{\mathrm{d}}$ & \\
\hline \multicolumn{4}{|l|}{ Eating/drinking } \\
\hline Baseline & $56.78 \pm 19.45$ & $59.01 \pm 16.74$ & $0.541^{\mathrm{e}}$ \\
\hline Week 4 & $71.46 \pm 19.11$ & $74.04 \pm 20.47$ & \\
\hline Change $^{\mathrm{a}}$ & $14.68 \pm 18.98$ & $15.02 \pm 20.22$ & $0.909^{\mathrm{b}}$ \\
\hline In group P-value & $<0.001^{\mathrm{c}}$ & $<0.001^{\mathrm{d}}$ & \\
\hline \multicolumn{4}{|l|}{ Knowledge/control } \\
\hline Baseline & $46.51 \pm 17.72$ & $51.15 \pm 15.68$ & $0.145^{\mathrm{b}}$ \\
\hline Week 4 & $61.34 \pm 18.00$ & $64.92 \pm 18.00$ & \\
\hline Change $^{\mathrm{a}}$ & $14.83 \pm 20.28$ & $13.78 \pm 17.62$ & $0.783^{\mathrm{e}}$ \\
\hline In group $P$-value & $<0.001^{\mathrm{d}}$ & $<0.001^{\mathrm{d}}$ & \\
\hline \multicolumn{4}{|l|}{ Work/study } \\
\hline Baseline & $57.68 \pm 17.26$ & $59.75 \pm 17.49$ & $0.553^{\mathrm{e}}$ \\
\hline Week 4 & $68.38 \pm 20.26$ & $72.05 \pm 19.71$ & \\
\hline Change $^{\mathrm{a}}$ & $10.70 \pm 20.58$ & $12.30 \pm 20.11$ & $0.984^{b}$ \\
\hline In group $P$-value & $<0.001^{\mathrm{c}}$ & $<0.001^{\mathrm{d}}$ & \\
\hline
\end{tabular}

NDI-K, Nepean Dyspepsia Index-Korean version.

${ }^{a}$ Changes in score related to symptoms (week 4-baseline).

${ }^{\mathrm{b}}$ Two-sample $t$ test.

'Wilcoxon signed rank test.

${ }^{\mathrm{d}}$ Paired $t$ test.

${ }^{\mathrm{e}}$ Wilcoxon rank sum test. sion/sleep, interference with daily activities, eating/drinking, knowledge/control, and work/study, increased significantly and there were no significant differences between the 2 groups (Table 5 ).

\section{Other efficacy analyses}

The changes in GIS scores from baseline to weeks 2 and week 4 for exploratory efficacy variables according to $H$. pylori status (Supplementary Table 2) and FD subtype (Supplementary Table 3) did not differ between the 2 groups. In addition, there were no differences between the 2 groups in terms of changes in GIS scores for the various symptoms, improvement rates in global assessment, and changes in the NDI-K for the total and for each subscale according to $H$. pylori status and FD subtype.

\section{Safety}

There were no differences in quantity and time of drug exposure between the mosapride citrate SR and mosapride citrate CR groups (95.7\%, vs $97.8 \%, P=0.127)$. The compliance between the 2 groups was not different $(98.0 \%$ vs $92.0 \%, P=0.206)$. Treatmentrelated adverse events were reported by 12 patients $(20.0 \%)$ in the mosapride citrate SR group, and 8 (13.6\%) in the mosapride citrate CR group: 2 in the mosapride citrate SR and 1 in the mosapride citrate $\mathrm{CR}$ group discontinued treatment due to adverse events. There were 3.3\% (2/60, 4 cases) drug adverse reactions in the mosapride citrate SR group: nausea, flatulence, and increase in triglycerides, and $6.8 \%$ (4/59, 7 cases) in the mosapride citrate CR: nausea, abdominal discomfort, constipation, diarrhea, vomiting, and edema $(P=0.439)$. No serious adverse events or adverse drug reactions were reported in either group. Numbers of red blood cells, hemoglobin, and hematocrit decreased significantly in the mosapride citrate SR group but the extents of the decreases did not differ from those in the mosapride citrate CR group. Although there were significant changes in triglycerides, phosphorus, blood urea nitrogen, creatinine, alkaline phosphatase, and total bilirubin, those changes had no clinical significance.

\section{Discussion}

The present study was conducted to evaluate the non-inferiority of mosapride citrate SR once daily to mosapride citrate $\mathrm{CR}$ 3 times a day for managing FD patients with moderate dyspeptic symptoms. This multi-center, randomized, double-blind, activecontrolled, non-inferiority trial showed that 4-week treatment with mosapride citrate SR once daily improved GIS scores, patientreported global assessments of GIS, and dyspepsia-related QoL, 
without significant differences from mosapride citrate CR 3 times a day. Mosapride citrate SR also had a similar safety profile to mosapride citrate $\mathrm{CR}$, with no serious adverse events. These results clearly demonstrate the non-inferiority of mosapride citrate SR once daily compared with mosapride citrate CR 3 times a day, in terms of efficacy and safety.

The efficacy of once daily mosapride citrate SR in treating GIS in FD patients was confirmed by the results that the change in GIS scores from baseline was $-10.04 \pm 4.45$ after a 4 -week treatment with mosapride citrate SR, compared with $-10.86 \pm 5.53$ for mosapride citrate CR 3 times a day. The mean difference in GIS changes between the 2 groups was 0.82 (95\% CI, $-1.17,2.81$ ), demonstrating non-inferiority of mosapride citrate SR to mosapride citrate $\mathrm{CR}$ since the upper limit of $\mathrm{CI}$ was below 3 .

Mosapride citrate SR is a sustained-release tablet that contains 2 components, a rapid-releasing component, and a slow-releasing component. Mosapride citrate SR once a day has similar pharmacokinetic characteristics to mosapride citrate CR 3 times a day. A test in healthy volunteers demonstrated biological equivalence between the 2 drugs by showing that the area under the curve of blood levels-time for mosapride citrate SR was almost equal to that for mosapride citrate CR 3 times a day. Moreover, the blood concentration of mosapride citrate SR was maintained above the threshold of effectiveness for more than 14 hours from morning to night in contrast to the see-saw fluctuations for mosapride citrate CR 3 times a day. The active constituent of mosapride citrate SR, mosapride, is a 5-hytroxytryptamine 4 receptor agonist that is known to increase antral contraction and fundic relaxation and thereby accelerate gastric emptying. ${ }^{5,6}$ Improvement of gastric emptying by mosapride was shown in patients with interferon-induced gastroparesis. ${ }^{15}$ Not only gastric emptying but also small intestinal transit was found to be improved in a study of patients undergoing capsule endoscopy tests showing a higher rate of test completion by mosapride. ${ }^{16}$ This increased gastric activity seems to be due to neuronal reconstruction induced by mosapride. ${ }^{17,18}$

In the Korean guidelines for $\mathrm{FD}$, prokinetics like mosapride, a main constituent of mosapride citrate $\mathrm{CR}$, are recommended for managing symptoms in FD patients. ${ }^{9}$ Mosapride citrate $\mathrm{CR}$ is currently used in many patients with various dyspeptic symptoms as well as in FD. Mosapride has also been proven to be effective and safe in previous clinical studies of $\mathrm{FD}$ patients. It was more effective in improving FD symptoms than domperidone, the most common comparator prokinetic, ${ }^{19}$ although there was inconsistency in studies comparing with placebo. ${ }^{20,21}$ In studies of patients with FD, upper abdominal distension as well as total GIS scores responded much better to mosapride $5 \mathrm{mg} 3$ times a day than to domperidone $10 \mathrm{mg}$ 3 times a day. Furthermore, mosapride was as effective or more effective than acid-suppressive agents or mucosal protectants. In studies comparing it with a potent acid-suppressive drug, proton pump inhibitor, mosapride showed similar efficacy in FD patients. ${ }^{22,23}$ In a large-scale randomized clinical trial including more than 600 patients, after 2-week treatment with mosapride or teprenone, more patients preferred mosapride to teprenone. ${ }^{24}$ However, some investigators have questioned the effectiveness of prokinetics in $\mathrm{FD}$. In a meta-analysis of 13 randomized clinical trials published in 2015, mosapride was reported to be ineffective in FD compared with placebo or other drugs. ${ }^{25}$ Nonetheless, as the authors concluded, the inconsistency might be due to a variety of factors such as publication bias, lack of consistent diagnostic criteria, and different assessment outcomes.

The present study compared the efficacy of mosapride citrate $\mathrm{SR}$ and mosapride citrate $\mathrm{CR}$ by analyzing multiple variables including patient-reported global assessments of symptom improvement and dyspepsia-specific QoL as well as GIS scores. In terms of these secondary efficacy variables, there were no differences between the groups. First, our results showed that the change in GIS from baseline to week 2 was not different, suggesting rapid improvement of dyspepsia symptoms in the study patients. Also, the changes in GIS by week 4 were higher than by week 2 . Taken together, these findings indicate that mosapride citrate $\mathrm{SR}$ was able to improve FD symptoms in a short time, with increased efficacy over a longer period of time. Second, the study patients reported subjective symptom improvement. Patient-reported global symptom also improved similarly by weeks 2 and 4 in both groups. Third, dyspepsia-specific QoL also improved after 4 weeks of mosapride citrate SR and mosapride citrate $\mathrm{CR}$ treatment. Previously, it was reported that $\mathrm{QoL}$ of $\mathrm{FD}$ patients seldom improved in response to prokinetics. In a recent meta-analysis evaluating the effect of several prokinetics on FD patients, only itopride improved QoL compared with placebo. ${ }^{26}$ However, we found that total scores in NDI-K, as well as scores in each individual area of QoL, increased significantly in both groups by week 4 , although they were not compared directly with placebo.

We also conducted subgroup analyses according to $H$. pylori infection as well as FD subtypes in view of the fact that the pathophysiology FD involves complex factors including $H$. pylori infection, dysmotility, and hypersensitivity to acid or foods. In these subgroup analyses, we again found no significant differences between mosapride citrate $\mathrm{SR}$ and mosapride citrate $\mathrm{CR}$ groups: outcomes for change in GIS, global symptom improvement rates, and dyspepsia-specific QoL, from baseline to weeks 2 and 4 were 
similar regardless of $H$. pylori infection and subtype of FD. Symptom improvement rate (63.2\%) in patients with PDS subtype was higher than that in the whole group (54.9\%), as the previous study ${ }^{27}$ confirmed that mosapride use was more effective in patients with PDS subtype than in EP subtype or overlap subtype patients. All these results indicate that the efficacy of mosapride citrate SR once daily is similar to mosapride citrate CR 3 times a day.

There are some limitations to the present study. First, one of the most important advantages of mosapride citrate SR is a better compliance by avoiding having to take the medication several times a day. However, due to the need for blinding, this advantage could not be demonstrated in the study. Additionally, the present study did not compare the cost-effectiveness of the 2 drugs, although oncedaily administration would certainly reduce the cost. Second, since functional tests for gastric motility such as gastric emptying time was not used, we may not have excluded some patients with gastroparesis. To some extent, however, this was avoided by excluding patients who took drugs that could affect gastrointestinal motility, those with organic causes of gastroparesis or uncontrolled diabetes mellitus. Third, there was no placebo group for evaluating the efficacy of the study drugs. Metoclopramide, trimebutine, mosapride, domperidone, and proton pump inhibitors are all known to improve symptoms in patients with FD. Comparisons with other comparators might also be needed to validate the usefulness of mosapride citrate SR. Fourth, the study period was only about one month. However, most previous studies also compared symptom improvement after 4 weeks, although others examined symptom improvement after 1 to 2 or 6 to 8 weeks. Given that there was greater improvement of symptoms after 4 weeks than 2 weeks, it may be necessary to compare symptom improvement rates over longer times. Finally, the number of study patients was relatively small although the number recruited was appropriate after taking into account dropout rates. Numbers of patients were particularly small in the subgroup analyses according to $H$. pylori status and FD subtype.

Despite these limitations, this study analyzed the efficacy of mosapride citrate SR in patients with FD in terms of multiple variables, namely GIS score, patient-reported global assessment of symptom improvement, and dyspepsia-specific QoL. In addition to the variety of endpoints, the study has merit in terms of study patients and duration. The enrolled patients had FD symptoms of more than moderate degree and follow-up was for 4 weeks in contrast to previous studies in which some patients had mild symptoms and follow-up was for only 1-2 weeks. In conclusion, mosapride citrate SR once daily for 4 weeks is as effective as mosapride citrate CR 3 times a day in improving GIS in FD patients and has a simi- lar safety profile. It also improves patient-reported global assessments of GIS and dyspepsia-specific QoL and might achieve better compliance than mosapride citrate $\mathrm{CR}$.

\section{Supplementary Materials}

Note: To access the supplementary tables mentioned in this article, visit the online version of Journal of Neurogastroenterology and Motility at http://www.jnmjournal.org/, and at https://doi. org/10.5056/jnm20061.

Financial support: Clinical trials were sponsored and conducted by Daewoong Pharmaceuticals Company Ltd.

Conflicts of interest: None.

Author contributions: Oh Young Lee, Myung-Gyu Choi, Hyunsoo Chung, Suck-Chei Choi, Nayoung Kim, Hyojin Park, In-Kyung Sung, Chong Il Sohn, Sam Ryong Jee, Jae Young Jang, Poong-Lyul Rhee, Moo In Park, Joong Goo Kwon, Kyung Sik Park, Kwang Jae Lee, and Joon Seong Lee contributed to the conception as well as study design along with data analysis and interpretation; Kang Nyeong Lee and Jin Hwa Park analyzed the data and wrote the manuscript; and Oh Young Lee performed critical review of the manuscript. Lastly, all authors gave a final approval for this version of the article to be published.

\section{References}

1. Ford AC, Marwaha A, Sood R, Moayyedi P. Global prevalence of, and risk factors for, uninvestigated dyspepsia: a meta-analysis. Gut 2015;64:1049-1057.

2. Masuy I, Van Oudenhove L, Tack J. Review article: treatment options for functional dyspepsia. Aliment Pharmacol Ther 2019;49:1134-1172.

3. Hiyama T, Yoshihara M, Matsuo K, et al. Meta-analysis of the effects of prokinetic agents in patients with functional dyspepsia. J Gastroenterol Hepatol 2007;22:304-310

4. Pittayanon R, Yuan Y, Bollegala NP, et al. Prokinetics for functional dyspepsia: a systematic review and meta-analysis of randomized control trials. Am J Gastroenterol 2019;114:233-243.

5. Odaka T, Suzuki T, Seza A, Yamaguchi T, Saisho H. [Serotonin 5-HT receptor agonist (mosapride citrate).] Nihon Rinsho 2006;64:1491-1494. [Japanese]

6. Kusunoki H, Haruma K, Hata J, et al. Efficacy of mosapride citrate in proximal gastric accommodation and gastrointestinal motility in healthy volunteers: a double-blind placebo-controlled ultrasonographic study. J Gastroenterol 2010;45:1228-1234

7. Kim DK. Effects of mosapride on upper gastrointestinal symptoms and gastric emptying in patients with functional dyspepsia. Korean J Neuro- 
gastroenterol Motil 2004;10:27-34.

8. Cho YK, Choi MG, Kim SH, et al. [The effect of mosapride on quality of life in functional dyspepsia.] Korean J Gastroenterol 2004;43:160-167. [Korean]

9. Jee SR, Jung HK, Min BH, et al. [Guidelines for the treatment of functional dyspepsia.] Korean J Gastroenterol 2011;57:67-81. [Korean]

10. Adam B, Liebregts T, Saadat-Gilani K, Vinson B, Holtmann G. Validation of the gastrointestinal symptom score for the assessment of symptoms in patients with functional dyspepsia. Aliment Pharmacol Ther 2005;22:357-363.

11. Talley NJ, Verlinden M, Jones M. Validity of a new quality of life scale for functional dyspepsia: a United States multicenter trial of the Nepean dyspepsia index. Am J Gastroenterol 1999;94:2390-2397.

12. Madisch A, Melderis H, Mayr G, Sassin I, Hotz J. [A plant extract and its modified preparation in functional dyspepsia. Results of a double-blind placebo controlled comparative study.] Z Gastroenterol 2001;39:511517. [German]

13. Rösch W, Vinson B, Sassin I. A randomised clinical trial comparing the efficacy of a herbal preparation STW 5 with the prokinetic drug cisapride in patients with dysmotility type of functional dyspepsia. Z Gastroenterol 2002;40:401-408.

14. Madisch A, Holtmann G, Mayr G, Vinson B, Hotz J. Treatment of functional dyspepsia with a herbal preparation. A double-blind, randomized, placebo-controlled, multicenter trial. Digestion 2004;69:45-52.

15. Kawamura E, Enomoto M, Kotani K, et al. Effect of mosapride citrate on gastric emptying in interferon-induced gastroparesis. Dig Dis Sci 2012;57:1510-1516.

16. Ida $\mathrm{Y}$, Hosoe N, Imaeda $\mathrm{H}$, et al. Effects of the oral administration of mosapride citrate on capsule endoscopy completion rate. Gut Liver 2012;6:339-343.

17. Matsuyoshi H, Kuniyasu H, Okumura M, et al. A 5-HT $\mathrm{H}_{4}$-receptor activation-induced neural plasticity enhances in vivo reconstructs of enteric nerve circuit insult. Neurogastroenterol Motil 2010;22:806-813, e226.

18. Tsuchida Y, Hatao F, Fujisawa M, et al. Neuronal stimulation with 5-hydroxytryptamine 4 receptor induces anti-inflammatory actions via alpha7nACh receptors on muscularis macrophages associated with postoperative ileus. Gut 2011;60:638-647.

19. Chen SY, Wang JY, Zhu CW, et al. [A randomized controlled Multicenter clinical trial on mosapride in the treatment of functional dyspepsia.] Zhonghua Liu Xing Bing Xue Za Zhi 2004;25:165-168. [Chinese]

20. Lin J, Ren M, Peng X, Xiao Y, Wang S, Yang L. Short-term efficacy of mosapride dispersible tablet on postprandial distress syndrome. Chin J Gastroenterol 2009;14:488-490.

21. Hallerbäck BI, Bommelaer G, Bredberg E, et al. Dose finding study of mosapride in functional dyspepsia: a placebo-controlled, randomized study. Aliment Pharmacol Ther 2002;16:959-967.

22. Hsu YC, Liou JM, Yang TH, et al. Proton pump inhibitor versus prokinetic therapy in patients with functional dyspepsia: is therapeutic response predicted by Rome III subgroups? J Gastroenterol 2011;46:183-190.

23. Jian Q, Ding X, Zhang S. Comparison of mosapride and pantoprazole in treating functional dyspepsia. Chin J Gastroenterol 2011;16:547-550.

24. Hongo M, Harasawa S, Mine T, et al. Large-scale randomized clinical study on functional dyspepsia treatment with mosapride or teprenone: Japan mosapride mega-study (JMMS). J Gastroenterol Hepatol 2012;27:62-68.

25. Bang CS, Kim JH, Baik GH, et al. Mosapride treatment for functional dyspepsia: a meta-analysis. J Gastroenterol Hepatol 2015;30:28-42.

26. Pittayanon R, Yuan Y, Bollegala NP, Khanna R, Leontiadis GI, Moayyedi P. Prokinetics for functional dyspepsia. Cochrane Database Syst Rev 2018;10:CD009431.

27. Yamawaki H, Futagami S, Wakabayashi M, et al. Management of functional dyspepsia: state of the art and emerging therapies. Ther Adv Chronic Dis 2018;9:23-32. 